\title{
Regularization tools for binary interactions
}

\author{
Sverre J. Aarseth \\ Institute of Astronomy, University of Cambridge, Cambridge, UK
}

\begin{abstract}
We first discuss two-body and chain regularization methods for direct N-body simulations on HARP-2 and GRAPE-6. The former is used for accurate integration of perturbed binaries and hierarchies, whereas the latter deals with strong interactions involving binaries. Combined with a powerful stability criterion for hierarchical systems, this versatile treatment provides an efficient way of studying all dynamical processes in globular clusters containing a realistic population of primordial binaries. These algorithms are also ideal for modelling a variety of astrophysical effects, such as averaging over Kozai cycles, tidal circularization, spin-orbit coupling and stellar collisions, where well-defined elements are used to describe near-singular solutions. We also describe a new time-transformed leapfrog scheme which has been developed to deal with black-hole binaries in galactic centres. This formulation is valid for large mass ratios and dominant two-body motions in a compact subsystem can be treated accurately in the post-Newtonian approximation. This method has been combined with the existing regularization algorithms into a new simulation code $N B O D Y 7$. Some preliminary results of a test problem illustrating possible applications are presented.
\end{abstract}

\section{Introduction}

The presence of binaries in star cluster simulations poses many technical questions which must be overcome in order to obtain satisfactory results. In the following, we review the main algorithms for studying a variety of interactions, ranging from physical collisions to stable hierarchies. It is somewhat paradoxical that the degree of difficulty in dealing with these two situations is such that the former is by far the easiest. Given a significant binary fraction, it is necessary to treat close encounters of three or more strongly interacting particles. This is achieved by three-body and chain regularization. In addition, stable configurations of three or more members form by various processes. These hierarchical structures range from triples to sextuplets. Upon ascertaining stability, such systems are described by two-body regularization. Here the inner binary may be subject to induced eccentricity increase due to the inclination effect, which can also be considered. With all these tools, it is now possible to study the long-term evolution of conventional star clusters, taking account of all relevant astrophysical processes. However, the modelling of black-hole binaries reveal some inefficiencies during the advanced stages of evolution. A new method is presented for dealing with this challenging problem. 


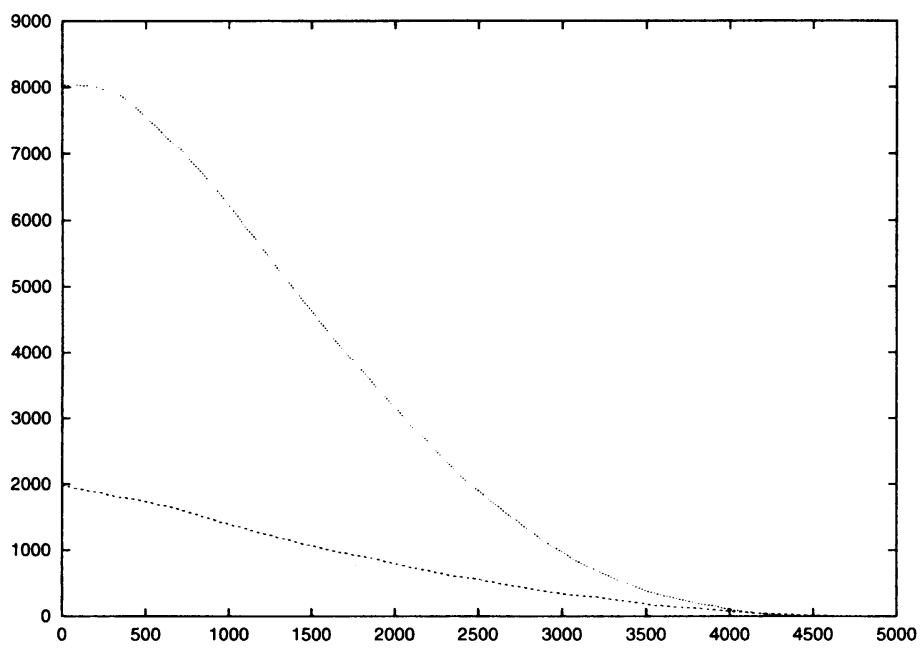

Figure 1. Number of single stars and binaries ( $N$-body units).

\section{Primordial Binaries}

Realistic star cluster simulations usually include a significant population of primordial binaries. We concentrate on hard binaries and choose a flat distribution of semi-major axes (Kroupa 1998). These binaries are integrated by the classical Kustaanheimo-Stiefel (1965, hereafter KS) regularization method. After trying several alternatives, the Stumpff Hermite formulation appears to be superior (Mikkola \& Aarseth 1998) and yields high accuracy at small perturbations. The main idea here is that correction terms are included for the neglected higher orders. In addition, the so-called slow-down procedure which exploits the adiabatic invariance (Mikkola \& Aarseth 1996) allows one KS orbit to represent several physical periods by scaling the perturbing force and physical time accordingly. At even smaller perturbations, the two-body orbit is assumed to be conserved over one or more periods, depending on the nearest neighbours. This approximation leads to a considerable speeding-up of the integration since the binary can now be treated as a single particle.

Use of regularized two-body descriptions facilitates implementation of astrophysical processes connected with finite-size effects (Aarseth 1996). Among these are tidal capture and circularization (Mardling \& Aarseth 2001), Rochelobe overflow, common-envelope evolution, magnetic or relativistic braking and physical collisions (Tout et al. 1997). In addition, slow expansion due to stellar mass loss or disruption following a neutron star velocity kick is also treated. An energy-conserving scheme is achieved by including a variety of correction procedures. Further details about the different processes have been reviewed elsewhere (Aarseth 1999a).

$\mathrm{KS}$ regularization has been implemented in several direct summation codes. Here we mention $N B O D Y 4$ for use on the special-purpose HARP-2 or GRAPE-6 computers and $N B O D Y 6$ which is suitable for laptops and workstations (Aarseth $1999 \mathrm{~b})$. The former is based on full $N$ force summation, whereas the latter em- 
ploys the Ahmad-Cohen (1973) neighbour scheme for increased efficiency. Both these recent versions use Hermite integration for the single particles (Makino 1991). With the special-purpose machines, the force on a binary centre of mass is also obtained by direct summation, followed by differential correction on the host due to the perturbers. Typical models of open clusters studied on HARP-2 consist of $N_{s}=8000$ single stars and $N_{b}=2000$ hard binaries, with total lifetimes exceeding 5 Gyr (cf. Hurley et al. 2001). Figure 1 shows the population of bound single stars and binaries as a function of time in a standard open cluster model. Near half-life at $t=1690$ (or $\simeq 2.2 \mathrm{Gyr}$ ), $N_{s}=4035$ and $N_{b}=957$; hence the binary population is not preferentially depleted.

\section{Hierarchical Systems}

Observations of the solar neighbourhood reveal a significant fraction of triples as well as systems of higher order. Likewise, analysis of star cluster models also show that such configurations are not rare (Aarseth \& Mardling 2001). The main channel for formation of triples is by binary-binary collisions and may be represented symbolically by

$$
B+B \Rightarrow[B, S]+S \text {. }
$$

This important process was already identified during scattering experiments where one single star is ejected (Mikkola 1983).

Depending on the orbital characteristics, an isolated triple may be stable and the life-time may still be considerable in the presence of perturbers. We test stability by a semi-analytical criterion for the outer pericentre given by (Mardling \& Aarseth 1999)

$$
R_{p}^{\text {crit }}=C\left[\left(1+q_{\text {out }}\right) \frac{\left(1+e_{\text {out }}\right)}{\left(1-e_{\text {out }}\right)^{1 / 2}}\right]^{2 / 5} a_{\text {in }}
$$

Here $C \simeq 2.8$ is a constant, $q_{\text {out }}$ and $e_{\text {out }}$ is the outer mass ratio and eccentricity, respectively, and $a_{\text {in }}$ the inner semi-major axis. Consequently, a triple system is said to be stable, provided $a_{\text {out }}\left(1-e_{\text {out }}\right)>R_{p}^{\text {crit }}$. This criterion has been checked extensively and appears to be quite robust. It has been generalized to the case of an outer binary and an inclination correction factor has also been included to represent the enhanced stability of retrograde orbits. The formation mechanism of quadruple systems is still under investigation but appears to be connected with multiple encounters. Here the early stage is characterized by a relatively soft binding energy, whereas many triples are already hard at the outset.

Once accepted as stable, a hierarchy is described by two-body motion and studied by $\mathrm{KS}$ regularization until the criterion is violated or the external perturbation dictates termination. In spite of the small formation rate, the population of hierarchical systems becomes significant after the early epoch, with typically a dozen members (Aarseth \& Mardling 2001). Temporary high-order systems of the type $[[B, S], S],[[B, S], B],[[B, B], B]$ occur and it is not uncommon even for quadruples to escape. Figure 2 illustrates the number of stable hierarchies as a function of time in the model used for Fig. 1. 


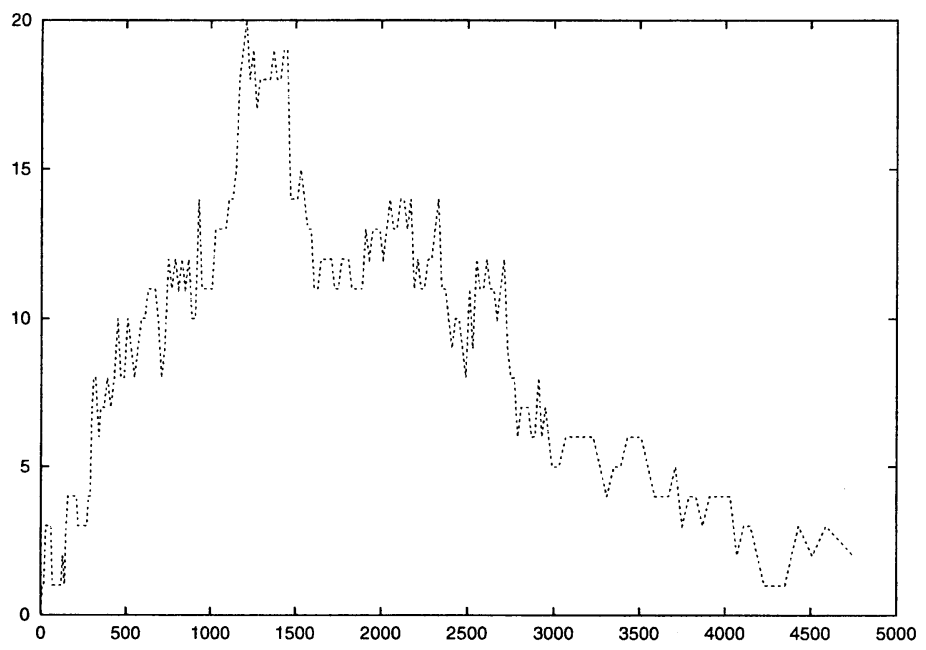

Figure 2. Stable hierarchical systems.

An attempt has been made to model the Kozai (1962) cycles of the inner binary for favourable values of the inclination (Mardling \& Aarseth 2001). Thus provided the maximum eccentricity reaches a sufficiently high value, tidal dissipation may be activated and initiate circularization with consequent orbital shrinking. We include the effect of stellar rotation (Hut 1981) which tends to advance the onset of Roche-lobe mass transfer by the synchronization process. The averaging treatment integrates the eccentricity and angular momentum vectors together with contributions from the outer body in the quadrupole approximation (Heggie 1996, Aarseth \& Mardling 2001). Here we also include apsidal motion and relativistic precession if relevant. The new two-body elements are converted to relative coordinates and velocities, followed by a transformation to $\mathrm{KS}$ variables for the inner binary which remains dynamically inert. In spite of various de-tuning effects, some examples of tidal circularization are in fact seen. Note that in order for this to occur, the eccentricity needs to reach quite high values; i.e. $e_{\max }>0.999$.

\section{Strong Interactions}

The presence of hard binaries invariably leads to close encounters involving large energy changes. Compact subsystems with 3-5 members are treated by chain regularization which also includes a slow-down procedure for super-hard binaries (Mikkola \& Aarseth 1993, 1996). In addition, unperturbed three-body regularization (Aarseth \& Zare 1974) is available during an ongoing chain interaction. Although there are only $\sim 10^{3}$ strong interactions during a typical cluster lifetime, many such events are associated with energetic ejections of single stars as well as binaries. Thus escape velocities $\sim 100 \mathrm{~km} \mathrm{~s}^{-1}$ may be seen in cluster models with rms velocity $\simeq 2 \mathrm{~km} \mathrm{~s}^{-1}$. 
Close binary-binary encounters often lead to the formation of triples, many of which are stable. In the alternative case of ejection, the remaining binary tends to acquire high eccentricity with possible collision. According to the results, the latter outcome occurs more frequently than hyperbolic two-body collisions, whereas tidal capture is seen on rarer occasions. The initiation of normal or chaotic tidal interactions is also connected with multiple encounters involving one or two binaries. Again all the astrophysical processes are treated using well-defined quantities. In the case of a physical collision, this requires careful iteration of the numerical solution which employs the Bulirsch-Stoer (1966) integrator. Here the osculating semi-major axis is obtained after evaluating the non-singular interactions, combined with the total subsystem energy.

Implementation of chain regularization in $N B O D Y 4$ and $N B O D Y 6$ requires considerable software development for decision-making. Here we need to combine the integration of regularized solutions based on non-linear time-steps with the Hermite scheme for commensurate steps. After nearly ten years experience, these procedures are now working quite well and are able to deal with most configurations. It is particularly important to identify stable hierarchies in order to enable a more efficient description. Since external perturbations are included, a long-lived system may cross the stability boundary slowly; hence frequent checks are needed.

The few-body regularization methods described above are also ideally suited to the study of small systems $(N \leq 10)$ as well as for classical scattering experiments. In particular, more simulations are needed to explore the parameter space for binary-binary scattering. However, depending on the termination conditions, it may be necessary to combine this approach with the variation of elements method or assume unperturbed two-body motion for weakly bound ejections (Mikkola 1983).

\section{Super-Massive Binary Method}

The regularization schemes discussed above work quite well for most stellar systems. However, the case of very large mass ratios requires special consideration in order to avoid the loss of efficiency and accuracy. The problem of black-hole binaries in galactic nuclei is topical and has received much attention. Direct integration on GRAPE-4 with $N=16,384$ and a component mass ratio of 256 yielded some interesting results (Makino \& Ebisuzaki 1996). Here the dominant interaction was calculated as point-masses, using full double precision on the host computer. The direct approach leads to difficulties for small pericentre distances and in any case this investigation did not describe extreme evolution. Attempts to use KS regularization have only been partially successful (Quinlan \& Hernquist 1997, Milosavljević \& Merritt 2001). One difficulty here is trapping of bound stellar orbits with respect to one of the massive binary components without resulting in coalescence. On general grounds, extension to chain regularization is also unlikely to be satisfactory because the total subsystem energy which appears explicitly in the equations of motion is dominated by the binary components. In view of the small period and large binding energy of such a binary, some other kind of regularization method is therefore desirable. 
In the following we describe a time-transformed leapfrog scheme which offers some practical advantages in dealing with large mass ratios (Mikkola \& Aarseth 2001). Consider first the standard leapfrog equations

$$
\begin{aligned}
\mathbf{r}_{\frac{1}{2}} & =\mathbf{r}_{0}+\frac{h}{2} \mathbf{v}_{0} \\
\mathbf{v}_{1} & =\mathbf{v}_{0}+h \mathbf{F}\left(\mathbf{r}_{\frac{1}{2}}\right) \\
\mathbf{r}_{1} & =\mathbf{r}_{\frac{1}{2}}+\frac{h}{2} \mathbf{v}_{1},
\end{aligned}
$$

where $h$ is the time-step and $\mathbf{F}$ denotes the acceleration at $t=\frac{1}{2} h$. We adopt a time transformation $d s=\Omega(\mathbf{r}) d t$, with $\Omega$ an arbitrary function and introduce a new auxiliary quantity $W=\Omega$. The new idea here is to evaluate $W$ by

$$
\dot{W}=\mathbf{v} \cdot \frac{\partial \Omega}{\partial \mathbf{r}} \text {. }
$$

This allows us to solve two sets of equations; namely (i) $\mathbf{r}^{\prime}=\mathbf{v} / W, t^{\prime}=1 / W$, and (ii) $\mathbf{v}^{\prime}=\mathbf{F} / \Omega, W^{\prime}=\dot{W} / \Omega$. Consequently, we write

$$
\begin{aligned}
\mathbf{r} & =\mathbf{r}_{0}+s \mathbf{v} / W \\
t & =t_{0}+s / W
\end{aligned}
$$

and

$$
\begin{aligned}
\mathbf{v} & =\mathbf{v}_{0}+\frac{s \mathbf{F}}{\Omega} \\
W & =W_{0}+s \frac{\mathbf{v}+\mathbf{v}_{0}}{2 \Omega} \cdot \frac{\partial \Omega}{\partial \mathbf{r}} .
\end{aligned}
$$

Here the solution may be combined into a form of leapfrog, taking $s=\frac{1}{2} h$ in (5); then $s=h$ in (6) and finally again $s=\frac{1}{2} h$ in (5).

The numerical solution is obtained by the Bulirsch-Stoer (1966) rational function extrapolation method. Thus, using the above leapfrog algorithm, several integrations are performed with gradually decreasing substeps $h$ and the results are extrapolated to zero steplength. This formulation has been generalized to include external perturbations of conservative type. An iterative procedure is necessary for treating the velocity-dependent relativistic corrections and the post-Newtonian approximation (PPN2.5) has been implemented. In either case, the energy of the subsystem is no longer constant but its change can be determined by straightforward integration.

Simple tests indicate that the time-transformed leapfrog (TTL) method is very promising. Thus significantly higher accuracy with about half the number of function evaluations was achieved when including the time transformation. This test employed the so-called Pythagorean three-body problem but it is desirable to examine other initial conditions. Moreover, reliable solutions for coalescence by gravitational radiation have been obtained involving one or both of the massive binary components. Since the Bulirsch-Stoer integrator is rather expensive when including many interactions, the new method is intended for treating a compact subsystem containing a massive binary and significant perturbers. Finally, we note that integration of the time transforming function $W$ ensures a more well-behaved solution than direct evaluation. 




Figure 3. Evolution of semi-major axes; $N=5000$.

\section{NBODY7: A New Simulation Code}

The TTL method has already been combined with the star cluster code NBODY6. In view of the many different procedures, it will be known as NBODY7. Consequently, all the standard regularization methods are still included for increased flexibility. The special treatment of a super-massive binary may begin with an existing small semi-major axis as provided by another calculation, possibly proceeded by a stage of KS regularization (cf. Milosavljević \& Merritt 2001).

In the following, we consider the more general case of two single massive members located at arbitrary positions with typical velocities. Depending on the mass ratio and particle number, the two massive bodies tend to lose kinetic energy by dynamical friction and spiral to the centre on the mass segregation time-scale (Hemsendorf, Sigurdsson \& Spurzem 2001). At some stage, the components will form a binary and continue to shrink by ejecting other particles from the core until the conditions are suitable for KS regularization. Hence selection for treatment by the TTL method is postponed until the semi-major axis, $a_{\mathrm{bh}}$, reaches the characteristic size for a hard binary. The reason for the delay is that the Bulirsch-Stoer integrator only requires a few steps during each Kepler period and this would cause prediction problems for neighbouring particles which tend to have somewhat smaller time-steps by the Hermite method.

The presence of a central subsystem requires special decision-making in order to maintain an appropriate membership. This entails perturber selection as well as increasing the subsystem membership in a similar way as in KS and chain procedures. Likewise, ejected subsystem particles must be removed and reinitialized as field particles. However, use of a fixed boundary is too simplistic since some particles may have their apocentre just outside the critical value, with nearly constant semi-major axis. Typically, field particles inside $50 a_{\mathrm{bh}}$ are selected as perturbers, with each one having a small effect because of the mass ratio. Moreover, particles inside $20 a_{\mathrm{bh}}$ are chosen as members of the 


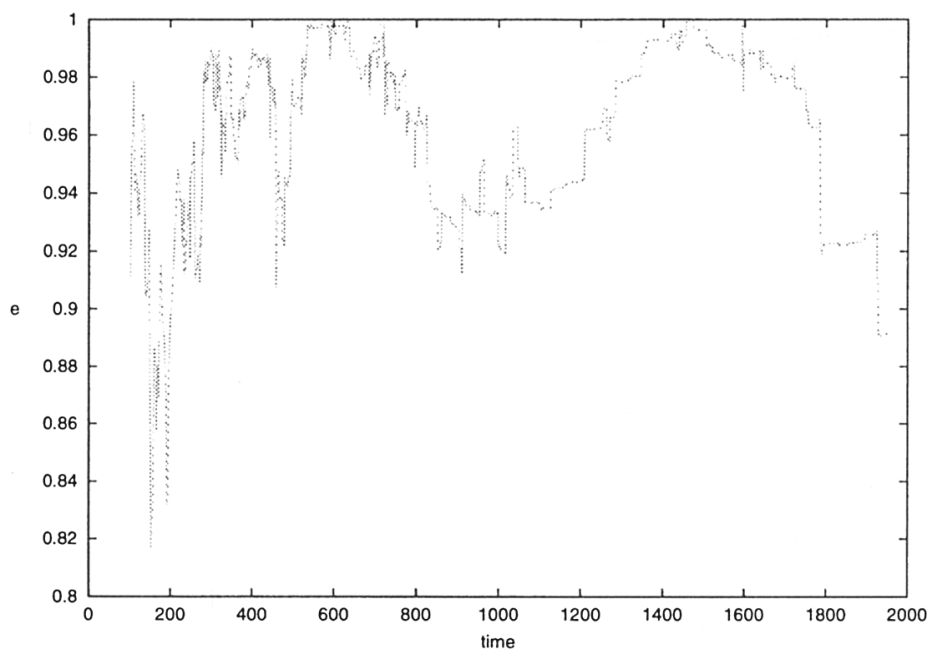

Figure 4. Eccentricity evolution.

subsystem itself. In order to prevent numerical problems, a softened potential may be adopted for interactions between the latter, whereas all terms involving the binary components are treated in the point-mass limit.

In the case of an ultra-hard binary containing black-hole components, the PPN2.5 post-Newtonian approximation is included for the two-body interaction. The full expression is given by (Soffel 1989)

$$
\mathbf{F}=\mathbf{F}_{0}+c^{-2} \mathbf{F}_{2}+c^{-4} \mathbf{F}_{4}+c^{-5} \mathbf{F}_{5},
$$

where $\mathbf{F}_{0}$ denotes the Newtonian force per unit mass and $c$ is the speed of light. Depending on circumstances, the other terms which are fairly time-consuming may be omitted. Alternatively, the relativistic radiation $\left(\mathbf{F}_{5}\right)$ may be included without contributions from the precession terms. There are two additional cases when the relativistic terms are considered. First, this is done if the osculating pericentre of the black-hole binary falls below a suitably small value related to the Schwarzschild radius. We note that the question of possible high eccentricity for realistic systems is still unresolved because of scaling uncertainties.

In the second critical case, the full GR treatment is activated if one of the subsystem members has a small impact parameter with respect to either component. Accordingly, a temporary switch from the dominant members is made to the new configuration, $m_{i}, m_{k}$, which would permit coalescence if relevant. Here we have adopted a provisional collision condition given by

$$
r_{\text {coll }}=6 G\left(m_{i}+m_{k}\right) / c^{2} .
$$

Numerical tests have been performed with artificially small values of $c$, demonstrating the correctness of the coalescence procedure.

A modest test calculation has been performed on a workstation in order to illustrate some features of the method. Initial conditions were chosen from a Plummer model containing $N=5000$ equal-mass particles, with the two first 


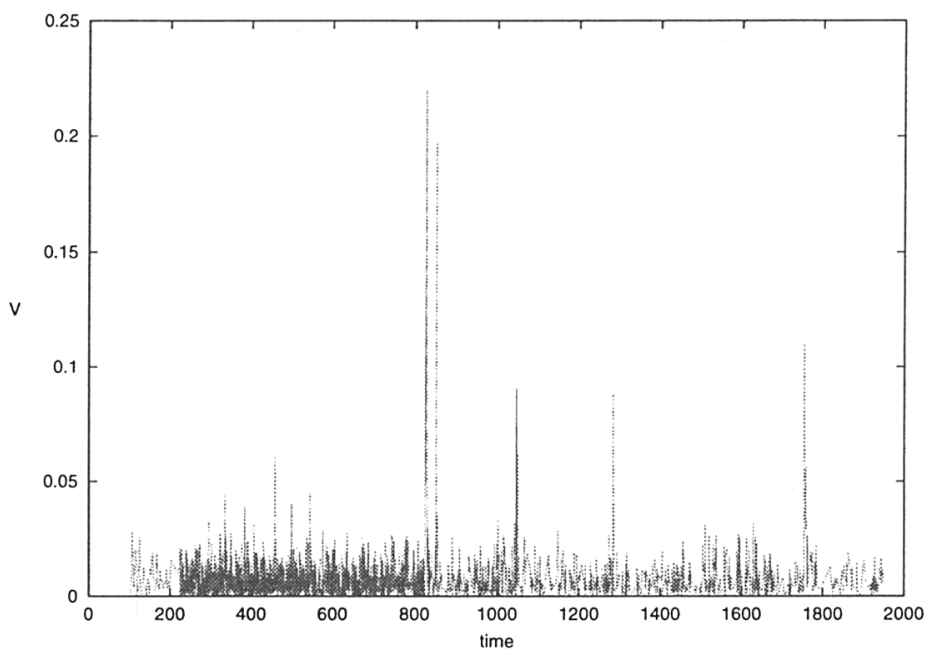

Figure 5. Square space velocity of massive binary.

being assigned intermediate masses $N^{1 / 2} \bar{m}$ as a good compromise. Figure 3 shows the evolution of the dominant binary during $2000 \mathrm{~N}$-body time units. A similar second example was also studied for comparison, again without any artificial scaling. In the first example, the KS solution was initialized at $a_{\mathrm{bh}}=$ $1.0 \times 10^{-3}$, followed by the TTL treatment from $a_{\mathrm{bh}}=3.8 \times 10^{-4}$ until $1.8 \times 10^{-5}$. The slow rate of shrinkage seen during the later stages is partly due to the depleted central density.

The behaviour of the eccentricity is displayed in Fig. 4. Two epochs of extremely high eccentricity can be seen. These maxima are associated with Kozai (1962) cycles (without GR) due to field particles in stable orbits with inclination near $90^{\circ}$. Depending on the density, such configurations will eventually be modified by external perturbations. Other trapped particles experience slingshot interactions and are ejected with large velocities. The corresponding recoil effect can be seen in Fig. 5 which illustrates the time evolution of the square space velocity. In particular, there are several spikes in addition to a number of relatively large values. Here the effective mass ratio is 140 , with an initial mean square velocity of 0.5 . Consequently, the resulting binary recoil leads to significant core wandering which has been noted before. As for possible losscone replenishments, we note that the central restoring force may also be weak in larger systems, thereby enabling significant excursions to take place.

\section{Conclusions}

The preceding sections have reviewed several regularization techniques which have proved themselves in star cluster simulations (cf. Hurley et al. 2001). Although some sophisticated decision-making is required for efficient use, such formulations improve the numerical accuracy and also enable many astrophysical processes to be studied by well-behaved variables. In the second part, we 
described a new method for treating a super-massive binary and its significant perturbers, with the addition of post-Newtonian terms where relevant. This formulation can be used to study compact subsystems in galactic nuclei. So far, the TTL scheme has been implemented in a star cluster code for workstations. Given the existing procedures, very little effort is required to combine it with the current $N B O D Y 4$ code. This timely development should allow much more realistic simulations on the powerful GRAPE-6 special-purpose computer.

\section{References}

Aarseth, S.J. 1996, in Dynamical Evolution of Star Clusters, ed. P. Hut \& J. Makino (Dordrecht: Kluwer), 161

Aarseth, S.J. 1999a, in Impact of Modern Dynamics in Astronomy, ed. J. Henrard \& S. Ferraz-Mello (Dordrecht: Kluwer), 127

Aarseth, S.J. 1999b, PASP, 111, 1333

Aarseth, S.J. \& Mardling, R.A. 2001, in Evolution of Binary and Multiple Star Systems, ed. P. Podsiadlowski, S. Rappaport, A. King, F. D'Antona \& L. Burderi (San Francisco: ASP), 77

Aarseth, S.J. \& Zare, K. 1974, Celes. Mech. 10, 185

Ahmad, A. \& Cohen, L. 1973, J. Comput. Phys. 12, 389

Bulirsch, R. \& Stoer, J. 1966, Num. Math. 8, 1

Heggie, D.C. 1996, personal communication

Hemsendorf, M., Sigurdsson, S. \& Spurzem, R. 2001, astro-ph/0103350

Hurley, J., Tout, C.A., Aarseth, S.J. \& Pols, O.R. 2001, MNRAS, 323, 630

Hut, P. 1981, A\&A, 99, 126

Kozai, Y. 1962, AJ, 67, 591

Kroupa, P. 1998, MNRAS, 298, 231

Kustaanheimo, P. \& Stiefel, E. 1965, J. Reine Angew. Math. 218, 204

Makino, J. 1991, PASJ, 43, 859

Makino, J. \& Ebisuzaki, T. 1996, ApJ, 465, 527

Mardling, R. \& Aarseth, S. 1999, in The Dynamics of Small Bodies in the Solar System, ed. B.A. Steves \& A.E. Roy (Dordrecht: Kluwer), 385

Mardling, R.A. \& Aarseth, S.J. 2001, MNRAS, 321, 699

Mikkola, S. 1983, MNRAS, 203, 1107

Mikkola, S. \& Aarseth, S.J. 1993, Celes. Mech. Dyn. Astron. 57, 439

Mikkola, S. \& Aarseth, S.J. 1996, Celes. Mech. Dyn. Astron. 64, 197

Mikkola, S. \& Aarseth, S.J. 1998, New Astron. 3, 309

Mikkola, S. \& Aarseth, S.J. 2001, Celes. Mech. Dyn. Astron. submitted

Milosavljević, M. \& Merritt, D. 2001, astro-ph/0103410

Quinlan, G.D. \& Hernquist, L. 1997, New Astron. 2, 533.

Soffel, M.H. 1989, Relativity in Astrometry, Celestial Mechanics and Geodesy (Berlin: Springer), p. 141

Tout, C.A., Aarseth, S.J., Pols, O.R., \& Eggleton, P.P. 1997, MNRAS, 291, 732 